J. Sustain. Wireless Syst.

Vol.02/ No. 1

Pages: 23-32

http://irojournals.com/irosws/

DOI: https://doi.org/10.36548/jsws.2020.1.003

\title{
Scheduled Optimal SDWSN Using Wireless Transfer of Power
}

\author{
Dr.C.ANAND, \\ ASSOC.PROF,DEPT OF CSE, \\ KSRCE,TIRUCHENCODE. \\ canand02@gmail.com
}

\begin{abstract}
Energy scarcity is one of the biggest threats faced by today's society because of the increasing demand for Wireless Sensor Networks. Wireless power transfer is one of the merging solutions that promise to address this energy scarcity and replenish the sensor nodes. In this method, dedicated energy transmitters are used to transfer energy to the sensor nodes. Moreover, Software-defined Wireless Sensor Networks (SDWSNs) have been proposed recently and are defined such that they are used to utilize the WSN resources to its maximum potential. The proposed methodology proposes a Wireless power transfer using SDWSN. We have designed a method to find the minimum energy transmitters by placing the energy transmitters in the node. A trade-off is made between fair distribution of energy and maximum energy charged in the network to place the energy transmitters. The proposed mechanism is defines a utility function in order to increase fairness and total energy charged. The goal is to decrease the energy consumed by the transmitters while retaining the charge of the sensor nodes. The simulation results illustrate the optimum efficiency of the proposed methodology with respect to energy consumption, number of tasks, number of energy transmitters, fairness and energy charged.
\end{abstract}

Keywords: wireless sensor networks, RF energy harvesting, optimization, optimal node placement

\section{Introduction}

Wireless Sensor Networks (WSNs) are an important part of many applications such as public safety network, environment monitoring, smart homes, health care, intelligent transport systems, smart grids etc. In order to improve the performance of WSNs, research has been done on sensors which are used to do particular tasks such as monitoring humidity, temperature, etc. But, there are different scenarios and requirements for modern WSN characterization. Taking this into consideration, it has been found that Software-defined WSNs are the apt solution to this issue [1]. There are many types of sensors which are embedded into the SDWSNs and the node can be configured such that it is designed for a specific sensing task. The biggest threat faced by the SDWSNs and WSNs is the limited battery life of the sensor node. Hence a number of energy efficient methods have been proposed over the years which are focused on reducing the energy consumption by the nodes in [2]-[5]. Survey shows that Wireless power transfer and RF energy harvesting are the best methods recommended to improve the lifetime of the networks in [6]-[9]. In the wireless power transfer method, in order to increase the WSNs lifetime, the external energy sources can be deployed intentionally into the surroundings [10]. Similarly, RF sources are used to harvest energy by the sensor nodes in RF energy harvesting, though there is no guarantee of ambient energy sources. Assumptions are made that the energy consumed by transmitters are increased due to 


\section{J. Sustain. Wireless Syst.}

Vol.02/ No. 1

Pages: 23-32

http://irojournals.com/irosws/

DOI: https://doi.org/10.36548/jsws.2020.1.003

periodic switching 'on' and 'off' of the energy sources. Wireless passive sensor networks are another methodology used to increase the WSNs lifetime with the help of RF sources externally. The modulated backscattering design in which antenna impedance of the sensor nodes switch and reflect the signal that arrives from the RF source to transmit data. They operate identical to wireless power transfer by assuming the role of more than one RF energy transmitters that transfer the energy without taking into account the demand of sensor nodes. The SDWSNs give a lot of flexibility to the sensor nodes for energy harvesting and charging based on the scenario and their requirement. Similar to the issues faced by the traditional WSNs, SDWSNs also face the problem of energy-efficiency. The energy consumed is a direct measure with respect to the active energy transmitters. Hence, lesser the number of transmitters used, lesser is the consumed energy level. But the sensor nodes that require charging many be located close or far from each other and will require energy charge prior to charging the other node. Hence the efficiency of the power transfer in SDWSNs need to be investigated in this regard. The proposed work considers a SDWSN with wireless power transfer that is built using energy transmitters, sensor nodes and controller as represented in the Figure 1.

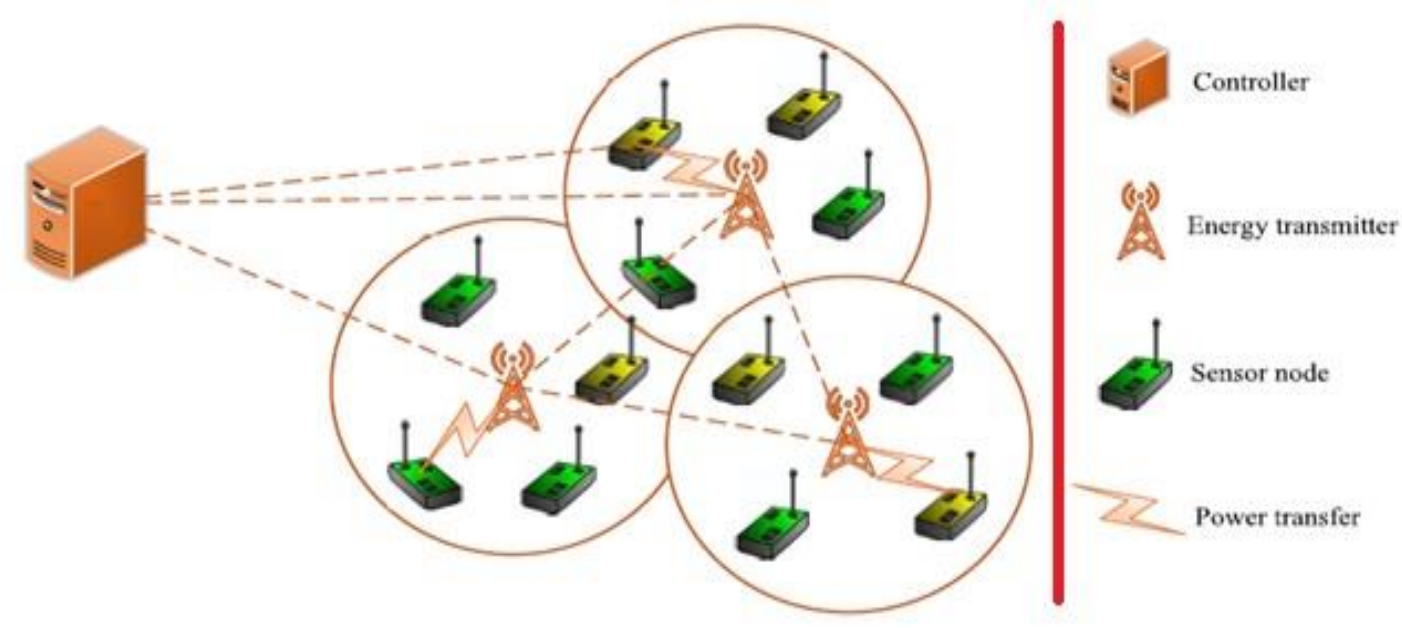

Figure 1. An illustration of SDWSNs with wireless power transfer.

The problem of energy efficient scheduling, minimum energy transmitter's requirement and optimal placement are addressed in this paper. Here the SDWSNs are used with Internet of Things (IoT) in order to further enhance network lifetime and improve energy efficiency. The major contributions of the proposed work are as listed below:

- Fair distribution of energy and joint maximization of energy charged is proposed for optimal placement mechanism such that it covers all the sensors within the range. 
J. Sustain. Wireless Syst.

Vol.02/ No. 1

Pages: 23-32

http://irojournals.com/irosws/

DOI: https://doi.org/10.36548/jsws.2020.1.003

- Second, on satisfying the minimum energy charging constraint of the sensor node, the number of energy transmitters required are calculated.

- Third, a scheduling scheme is proposed which can decrease the total energy consumed by individual energy transmitters.

- Finally, the proposed methodologies are validated using extensive simulations.

\section{Related Works}

A short overview about the SDWSNs are presented in this section and their recent works in wireless power transfer and energy harvesting in WSNs are also overviewed.

\section{A. Wireless Power Transfer and Energy Harvesting in WSN}

In [3] and [11] the energy harvesting schemes for WSNs are reviewed in detail. The authors in [20] have studied the RF energy harvesting feasibility and the observations are recorded for the RF power densities in the bands GSM 1800 and GSM 900. Based on the observations, it is observed that super capacitors are used to store the harvested energy to power the WSN. In [13], energy analysis and models of WSNs that operate with wireless power transfer are surveyed. Similarly in [14] and [15] the charging time and impact of energy transmitters are observed by the authors. In order to optimize the sensor nodes' energy sharing a MAC protocol has been designed and the performance is analyzed with RF energy harvesting in [16]. The performance evaluation takes into consideration the delay insensitive and sensitive scenarios. In [14], for wireless power transfer, power allocation and joint optimization of charger placement is used. Maximization of the charging quality is done using power allocation and location of the charger.

\section{B. Software-Defined Wireless Sensor Networks}

In order to decrease network management complexity, networking approach using predefined software is used by the WSNs. Some investigations have also been made regarding the feasibility of SDWSNs. [17] present technical challenges that were handled by the WSNs and SDNs. In [18], the author proposed an SDWSN which was designed to program the sensor nodes such that information transferred between the controller and sensor nodes were reduced. A prototype of SDWSN has been designed and implemented with offers benefits that overthrow the traditional WSN. In [19] a tiny SDN is proposed 
J. Sustain. Wireless Syst.

Vol.02/ No. 1

Pages: 23-32

http://irojournals.com/irosws/

DOI: https://doi.org/10.36548/jsws.2020.1.003

that outlines an SDWSN with multiple controllers and in [6] a sensor activation strategy which is energy efficient is presented.

\section{Proposed Work}

\section{A. Network Model}

Let us consider a SDWSN which has $\mathrm{N}_{\mathrm{E}}$ number of energy transmitters, $\mathrm{N}_{\mathrm{S}}$ number of nodes for wireless power transfer and a network size S. An assumption is made that there is random distribution of the nodes within the network area. Every time a request is made by the node for power transfer, it is known as a task t. The energy transmitter $e \in\{1,2, \ldots, N E\}$ is assigned multiple tasks, T. Assumption is made that there is only one wireless interface for all the nodes further ensuring that they operate in either charging or transmission mode. Here Pe is the transmit power of the energy transmitter e. Hence, if the harvesting range is within that of e, then the energy transmitter can transfer power to $t$ (the task) such that $t \in T$. This can be expressed as follows:

$$
\varphi_{e t}=\left\{\begin{array}{l}
1, \text { if the task } t \text { is in the harvesting range of } e \\
0, \text { otherwise }
\end{array}\right.
$$

The harvesting ranges will according to the task for the energy transmitters. When the location of the energy transmitter and the node has been collected by the collector, the value of $\varphi_{e t}$ is found out. The network control and management falls under the responsibility go the controller and it is also assumed that there is exchange of information between the network control and management with the controller before initiation of the transfer. The harvesting slot/ duration is represented as $\delta_{\mathrm{e}, \mathrm{H}}$ which is the time in which the energy transmitter is activated. The range of the harvesting duration varies between $\delta_{\mathrm{e}, \mathrm{Hmin}}$ to $\delta_{\mathrm{e}, \mathrm{Hmax}}$ based on the sensor node requirements. Hence the energy transmitter can be used in both sleep as well as active mode. In the active mode, energy consumption can be represented as:

$$
\xi_{\mathrm{e}, \mathrm{A}}=\left(\mathrm{P}_{\mathrm{HC}}+\mathrm{P}_{\mathrm{RF}}+\mathrm{P}_{\mathrm{RP}}+\mathrm{P}_{\mathrm{e}}\right) \times \delta_{\mathrm{e}, \mathrm{H}}
$$

Where $\mathrm{P}_{\mathrm{RF}}$ and $\mathrm{P}_{\mathrm{HC}}$ are the power consumption of RF module and hardware circuit. Similarly PRP is the power consumption during the exchange of packets between the controller and energy transmitter.

In the sleep mode, energy consumption is represented as $\xi_{\mathrm{e}, \mathrm{S}}$. Similarly, the i-th sensor node's data transmission slot duration is represented by $\delta_{\mathrm{i}, \mathrm{D}}$ 
J. Sustain. Wireless Syst.

Vol.02/ No. 1

Pages: 23-32

http://irojournals.com/irosws/

DOI: https://doi.org/10.36548/jsws.2020.1.003

Hence the energy consumed can be represented as $\xi_{\mathrm{i}, \mathrm{D}}$ which can be expressed as follows:

$$
\xi_{\mathrm{i}, \mathrm{D}}=\left(\mathrm{P}_{\mathrm{S}}+\mathrm{P}_{\mathrm{R}}+\mathrm{P}_{\mathrm{HC}}+\mathrm{P}_{\mathrm{RF}}+\mathrm{P}_{\mathrm{i}, \mathrm{D}}\right) \times \delta_{\mathrm{e}, \mathrm{D}}
$$

where $P_{R}$ and $P_{S}$ are the power consumed in processing and sensing of the sensor nodes and power is denoted as $P_{i, D}$.

\section{Energy Charging Model}

Assumption is made that the energy transmitters have the ability to transmit in all directions. Based on the propoagation properties of the environment, harvesting circuit and transmit power, the quantity of energy charged by the node will vary. Similarly, in terrestrial environment, varying size of the obstacles will result in scattering, refraction and reflection of signals thereby increasing the path loss in such environment when compared with free space. This loss can be modelled as:

$$
\mathrm{L}_{\mathrm{T}}(\mathrm{d})=\mathrm{L}_{0}\left(\mathrm{~d}_{0}\right)+10 \omega \log \left(\mathrm{d} / \mathrm{d}_{0}\right)+\mathrm{X}_{\mathrm{f}}
$$

Where $\mathrm{d} 0$ is the distance between the transmitter and ther reference location which has a path loss of L0(d0).

\section{Scheduling and Placement}

There are two main components which contribute to an energy-efficient wireless power transfer which are 1)scheduling of energy transmitters and 2)placement of energy transmitters.

\section{A. Scheduling of Energy Transmitters}

Three stages are considered for optimum efficiency of the energy transmitter:

\section{Stage I: Request for power transfer}




\section{J. Sustain. Wireless Syst.}

Vol.02/ No. 1

Pages: 23-32

http://irojournals.com/irosws/

DOI: https://doi.org/10.36548/jsws.2020.1.003

First, a request for power (RFP) packet is sent by the SDWSN nodes to the controller, when the residual energy is found to be below the threshold level $\xi$ Th. This energy threshold is fixed based on the minimum energy required for critical operation of the node. The RFP packet that is transmitted will hold the energy charging requirements, the controller's ID and the requesting node's ID. In the proposed work MAC protocol is adopted. When the pre-set threshold energy is crossed, an RFP is sent by the sensor node for instant charging of the node using access priority mechanism so that the nodes which have residual energy less than $\xi T h$. can be charged immediately before other nodes start to transmit data. The other nodes are frozen since data transmission cannot take place during this time.

\section{STAGE II: Energy Transmitter Activation Optimization}

On receiving the RPF packet, the controller will first calculate $\varphi$ et for every energy transmitter. Based on the formulated algorithm, it is possible to activate an energy transmitter to harvest energy only if the task $t$ is scheduled on the energy transmitter e and is within the harvesting range. The primary objective of this proposal is to decrease the number of active energy transmitters, thereby minimizing the total energy consumption. Moreover, the energy transmitters that are required for the task should have a minimum harvesting duration. Based on the energy charging requirements and the harvesting duration the scheduling program is called. Hence the scheduling algorithm considered for energy transmitters in SDWSNs are bound and branch algorithm. The grant for power transfer (GPT) packet is sent to the energy transmitters on activation of energy transmitters to go ahead with the power transfer process.

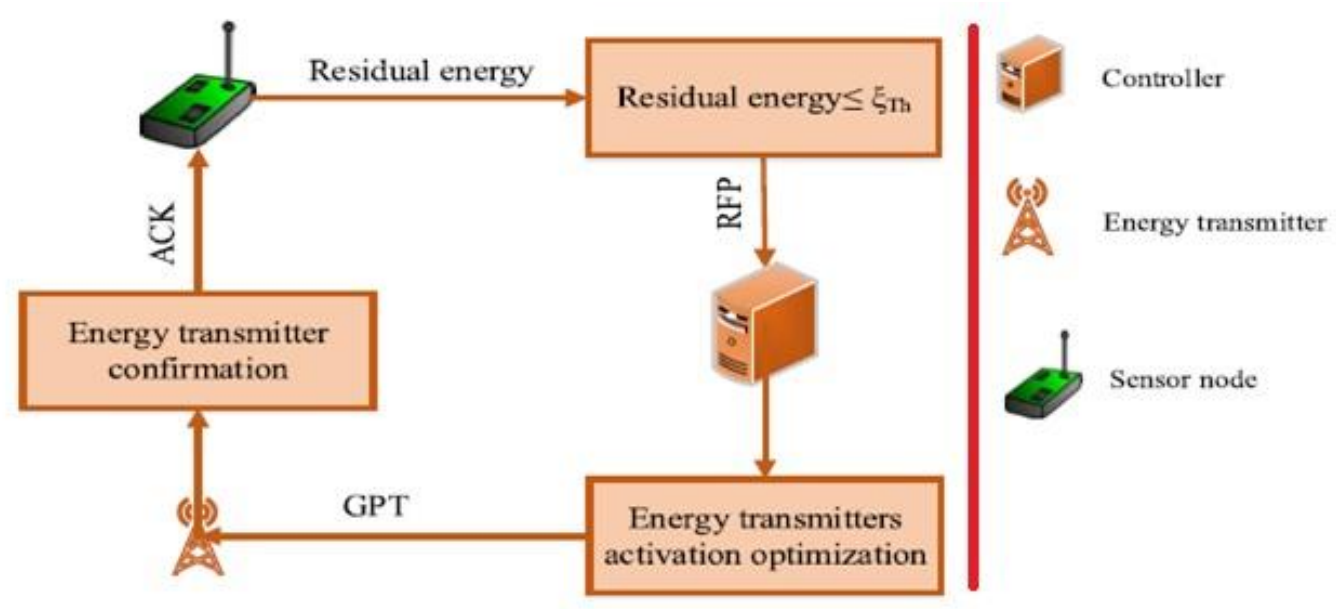

Figure 2. Stages of a MAC protocol in SDWSN 
J. Sustain. Wireless Syst.

Vol.02/ No. 1

Pages: 23-32

http://irojournals.com/irosws/

DOI: https://doi.org/10.36548/jsws.2020.1.003

\section{STAGE III: Energy Transmitter Configuration}

On receiving FPT packet, the energy transmitter will begin transmitter power to the node. An acknowledgement (ACK) is also sent by the energy transmitter and this packet will hold the information on duration of energy charging and central frequency of energy transmitter.

\section{Results and Discussion}

Using exhaustive search, the performance of bound and branch algorithm is done using wireless power transfer in SDWSNs. Energy consumption of the nodes in SDWSNs will depend on the requesting node's location. Random requests are generated for the sensor nodes and it is observed from Figure 3 that the for the proposed energy-efficient scheduling, the energy consumption isn't affected by the increase in energy transmitters. Hence it is concluded that only limited energy transmitters have to be activated by the SDWSNs controller.

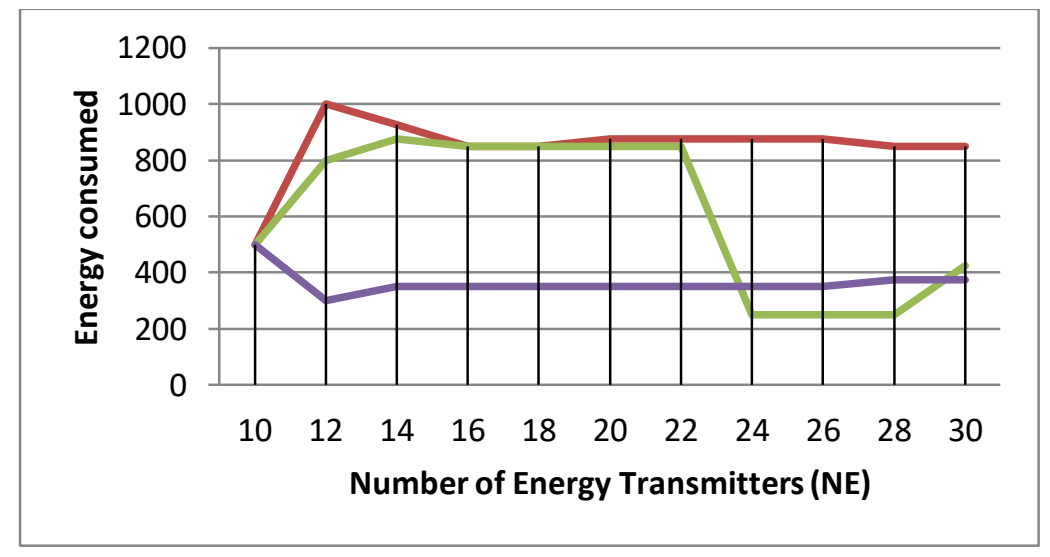

Figure 3 Number of Energy Transmitters Vs Energy Consumed for $\mathrm{T}=5$ and $\mathrm{T}=15$

Similarly Figure 5 shows a comparison of the number of energy transmitters vs energy consumption using the proposed algorithm. It is observed that as the network size increases, the energy consumed also increases. 
J. Sustain. Wireless Syst.

Vol.02/ No. 1

Pages: 23-32

http://irojournals.com/irosws/

DOI: https://doi.org/10.36548/jsws.2020.1.003

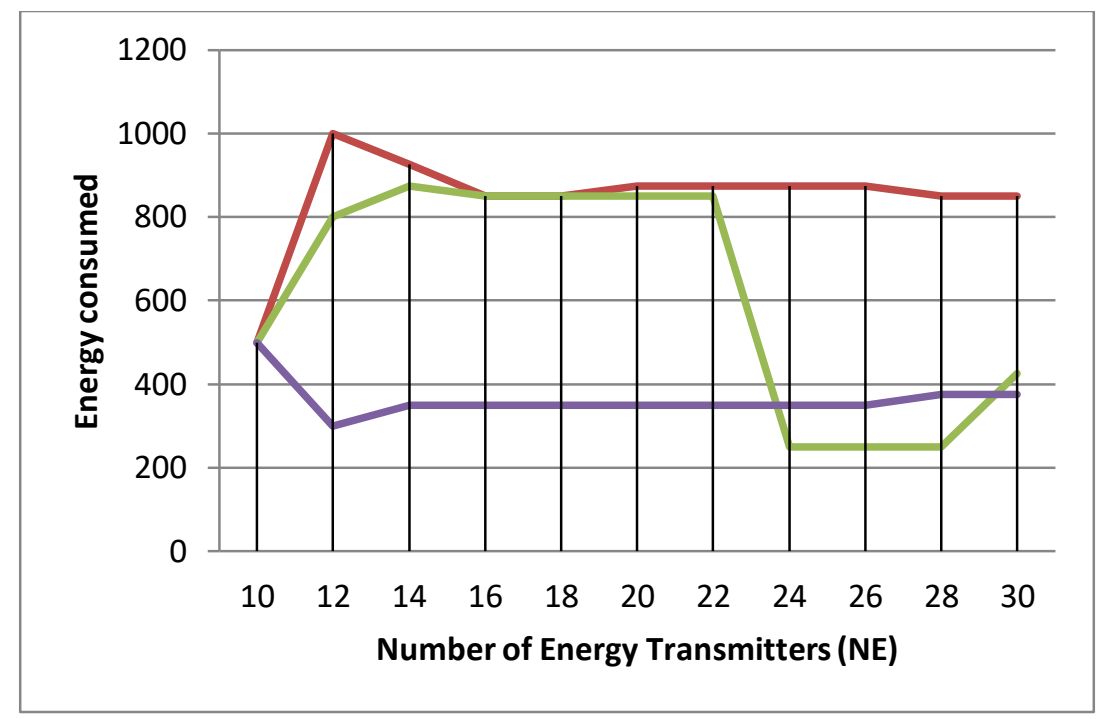

Figure 4. Number of Energy Transmitters Vs Energy Consumed for different network sizes

\section{Conclusion}

The ubiquitous applications and advancement in technology has shifted the paradigm of WSN to SDWSNs. The proposed work illustrates an optimal method of wireless power transfer which forms the underlying key technique towards optimizing SDWSNs. An energy-efficient scheduling algorithm is also proposed. This paper works on a binary integer linear programming problem which efficiently minimizes the energy consumed by the nodes while taking into consideration the energy harvested, harvesting duration and constraints on coverage. If has been found out that the scheduling of energy transmitters in SDWSNs will decrease the energy consumption of the nodes to a considerable amount using wireless power transfer. Future work in this paper can be directed towards overcoming path losses during energy transmission process. Similarly the use of variable transmission power with energy transmitters based on harvesting need will be a promising investigation.

\section{References}

[1] T. Luo, H.-P. Tan, and T. Q. S. Quek (Nov. 2012), "Sensor OpenFlow: Enablingsoftwaredefined wireless sensor networks,” IEEE Commun. Lett., vol. 16, no. 11, pp. 1896-1899.

[2] T. Rault, A. Bouabdallah, and Y. Challal, "Energy efficiency in wireless sensor networks: A top-down survey,” Comput. Netw., vol. 67, pp. 104-122, Jul. 2014. 
J. Sustain. Wireless Syst.

Vol.02/ No. 1

Pages: 23-32

http://irojournals.com/irosws/

DOI: https://doi.org/10.36548/jsws.2020.1.003

[3] W. Sun, Z. Yang, X. Zhang, and Y. Liu, "Energy-efficient neighbour discovery in mobile ad hoc and wireless sensor networks: A survey," IEEE Commun. Surveys Tuts., vol. 16, no. 3, pp. 1448-1459, 3rd Quart., 2014.

[4] A. A. Aziz, Y. A. Sekercioglu, P. Fitzpatrick, and M. Ivanovich, "A survey on distributed topology control techniques for extending the lifetime of battery powered wireless sensor networks," IEEE Commun. Surveys Tuts., vol. 15, no. 1, pp. 121-144, 1st Quart., 2013.

[5] W. Ejaz, G. A. Shah, N. U1 Hasan, and H. S. Kim, "Energy and throughput efficient cooperative spectrum sensing in cognitive radio sensor networks," Trans. Emerg. Telecommun. Technol., vol. 26, no. 7, pp. 1019-1030, Jul. 2015.

[6] A. S. M. Z. Kausar, A. W. Reza, M. U. Saleh, and H. Ramiah, "Energizing wireless sensor networks by energy harvesting systems: Scopes, challenges and approaches," Renew. Sustain. Energy Rev., vol. 38, pp. 973-989, Oct. 2014.

[7] X. Di, K. Xiong, Y. Zhang, and Z. Qiu, "Simultaneous wireless information and power transfer in two-hop OFDM decode-and-forward relay networks," KSII Trans. Internet Inf. Syst., vol. 10, no. 1, pp. 152-167, Jan. 2016.

[8] O. B. Akan, M. T. Isik, and B. Baykal, "Wireless passive sensor networks," IEEE Commun. Mag., vol. 47, no. 8, pp. 92-99, Aug. 2009.

[9] G. Uçtu, "Optimal transmission scheduling for energy harvesting systems and implementation of energy efficient scheduling algorithms on software defined radio," Ph.D. dissertation, Dept. Electr. Electron. Eng., Middle East Tech. Univ., Ankara, Turkey, Jun. 2014.

[10] L. Xie, Y. Shi, Y. T. Hou, and H. D. Sherali, "Making sensor networks immortal: An energyrenewal approach with wireless power transfer," IEEE/ACM Trans. Netw., vol. 20, no. 6, pp. 1748-1761, Dec. 2012.

[11] S. Sudevalayam and P. Kulkarni, "Energy harvesting sensor nodes: Survey and implications," IEEE Commun. Surveys Tuts., vol. 13, no. 3, pp. 443-461, 3rd Quart., 2011.

[12] T. B. Lim, N. M. Lee, and B. K. Poh, "Feasibility study on ambient RF energy harvesting for wireless sensor network," in Proc. IEEE Int. Microw. Workshop Ser. RF Wireless Technol. Biomed. Healthcare Appl. (IMWS-BIO), Singapore, Dec. 2013, pp. 1-3.

[13] M. Y. Naderi, K. R. Chowdhury, and S. Basagni, "Wireless sensor networks with RF energy harvesting: Energy models and analysis," in Proc. IEEE Wireless Commun. Netw. Conf. (WCNC), New Orleans, LA, USA, Mar. 2015, pp. 1494-1499.

[14] M. Y. Naderi, P. Nintanavongsa, and K. R. Chowdhury, "RF-MAC: A medium access control protocol for re-chargeable sensor networks powered by wireless energy harvesting," IEEE Trans. Wireless Commun., vol. 13, no. 7, pp. 3926-3937, Jul. 2014.

[15] P. Nintanavongsa, M. Y. Naderi, and K. R. Chowdhury, "A dual-band wireless energy transfer protocol for heterogeneous sensor networks powered by RF energy harvesting," in Proc. Int. Comput. Sci. Eng. Conf. (ICSEC), Nakhon Pathom, Thailand, Sep. 2013, pp. 387-392.

[16] T. Q. Wu and H. C. Yang, "On the performance of overlaid wireless sensor transmission with RF energy harvesting,” IEEE J. Sel. Areas Commun., vol. 33, no. 8, pp. 1693-1705, Aug. 2015.

[17] T. Luo, H.-P. Tan, and T. Q. S. Quek, "Sensor OpenFlow: Enabling software-defined wireless sensor networks,” IEEE Commun. Lett., vol. 16, no. 11, pp. 1896-1899, Nov. 2012. 
J. Sustain. Wireless Syst.

Vol.02/ No. 1

Pages: 23-32

http://irojournals.com/irosws/

DOI: https://doi.org/10.36548/jsws.2020.1.003

[18] L. Galluccio, S. Milardo, G. Morabito, and S. Palazzo, "SDN-WISE: Design, prototyping and experimentation of a stateful SDN solution for wireless sensor networks," in Proc. IEEE Conf. Comput. Commun. (INFOCOM), Hong Kong, Apr./May 2015, pp. 513-521.

[19] B. T. de Oliveira, C. B. Margi, and L. B. Gabriel, "TinySDN: Enabling multiple controllers for software-defined wireless sensor networks," in Proc. IEEE Latin-Amer. Conf. Commun. (LATINCOM), Cartagena, Colombia, Nov. 2014, pp. 1-6. 